How I do it

coloproctology $2020 \cdot 42: 331-338$

https://doi.org/10.1007/s00053-020-00457-6

Online publiziert: 22. April 2020

(c) Springer Medizin Verlag $\mathrm{GmbH}$, ein Teil von Springer Nature 2020

Die laparoskopische ventrale Netzrektopexie (LVR) erfreut sich seit ihrer Einführung durch D'Hoore und Penninckx 2004 großer Beliebtheit [1]. Initial für die Korrektur des externen Rektumvollwandprolapses vorgesehen, wurde die Indikation aufgrund der geringen Morbidität zunehmend erweitert, insbesondere um die Therapie des inneren Rektumprolapses (Intussuszeption) und der komplexen Rektozele, wenn diese mit funktionellen Einschränkungen im Sinne einer obstruktiven Defäkation (OD) oder einer fäkalen Inkontinenz (FI) vergesellschaftet sind.

Anders als bei anderen abdominell pexierenden Verfahren erfolgt bei der LVR keine posteriore Mobilisation des Rektums und damit eine Schonung des autonomen Nervensystems. Ebenso wird auf eine Resektion des Sigmas verzichtet, was zu der geringen Morbidität und den guten funktionellen Ergebnissen postoperativ beiträgt.

\section{) Die Indikationsstellung bedarf insbesondere bei funktioneller Entleerungsstörung großer Sorgfalt}

Aufgrund dessen ist die LVR zu dem am häufigsten angewandten Verfahren in Europa zur Behandlung des äußeren und inneren Rektumprolapses (Intussuszeption) avanciert $[2,3]$. Trotz der geringen Gesamtmorbidität bedürfen ca. 8-9\% der Patienten nach LVR eines Revisionseingriffs im Verlauf, welcher aufgrund der Netzimplantation im kleinen Becken und der konsekutiven Fibrosierung zu einer komplexen operativen Prozedur

\title{
Mia Kim
}

Klinik und Poliklinik für Allgemein-, Viszeral-, Transplantations-, Gefäß- und Kinderchirurgie, Universitätsklinikum Würzburg, Würzburg, Deutschland

\section{Laparoskopische ventrale Rektopexie}

führen kann $[4,5]$. Auch im Zuge der öffentlichen Diskussion über mögliche Langzeitfolgen nach Netzimplantationen im kleinen Becken, angestoßen vor allem von Patientenvertretern, wird nicht nur die urogynäkologische Netzanwendung, sondern auch die Indikation zur LVR in der internationalen koloproktologischen Gemeinde kritischer diskutiert. Dementsprechend bedarf die Indikationsstellung zur LVR insbesondere bei Vorliegen einer funktionellen Entleerungsstörung auf dem Boden von morphologischen Veränderungen wie Intussuszeption und Rektozele großer Sorgfalt.

\section{Indikation und Diagnostik}

Die Indikation zur LVR kann zur operativen Therapie des externen Rektumprolapses auf der einen Seite oder einer funktionellen Einschränkung im Sinne einer OD bei morphologischen Veränderungen wie einer Intussuszeption oder einer komplexen Rektozele auf der anderen Seite gestellt werden. Trotz der guten funktionellen Ergebnisse und der geringen Morbidität ist die Indikation zur LVR aufgrund der Fremdkörperimplantation im kleinen Becken streng zu stellen.

Liegt das klinische Bild eines externen Rektumvollwandprolapses vor, so sollte im Rahmen der präoperativen Diagnostik eine Koloskopie bei den zumeist betagten Patienten endoluminale Pathologien ausschließen.

Liegt kein externer Rektumvollwandprolaps vor, sondern eine funktionelle Enddarmentleerungsstörung wie die OD oder eine FI, so ist eine erweiterte, differenzierte Diagnostik notwendig. Die erweiterte koloproktologische Diagnostik ist von besonderem Stellenwert, da das Vorliegen einer Intussuszeption oder einer komplexen Rektozele per se keinen pathologischen Befund und damit keine Operationsindikation darstellt. Die relative Operationsindikation besteht nur bei einer OD/FI, die mit Einschränkung der Lebensqualität einhergeht und bei Vorliegen eines morphologischen Korrelats (Intussuszeption und/oder komplexe Rektozele), welche auf bereits durchgeführte konservative Therapiemaßnahmen refraktär ist. Bei benigner Grunderkrankung kommt somit der Darstellung des morphologischen Korrelats, die durch eine operative Prozedur korrigiert werden kann, dem Ausmaß der damit einhergehenden funktionellen Einschränkung und der Auswirkung auf die Lebensqualität des Patienten eine entscheidende Bedeutung zu.

Dabei ist zu beachten, dass das Ausmaß der morphologischen Veränderungen und das Ausmaß der funktionellen Einschränkungen zum einen nicht miteinander korrelieren müssen, und zum anderen, dass morphologische Veränderungen wie eine Intussuszeption oder Rektozele auch bei asymptomatischen Patienten vorliegen können [6,7]. Da das Vorliegen von Intussuszeption und/ oder Rektozele für sich alleine keine Operationsindikation darstellt, kann erst die ausführliche Anamnese ihre funktionelle Wirksamkeit im Sinne einer OD oder einer FI aufzeigen. Dabei hat sich die Anwendung von validierten Messinstrumenten bewährt, da sie die typischen Symptome einer OD abfragen und über das reine Vorliegen einer OD hinaus auch das Ausmaß der funktionellen Einschränkung mit einem Punktewert bemessen. Geeignet ist dafür beispielsweise der OD-spezifische 
Tab. 1 ODS-Score nach Altomare. (Mod. nach [8], nichtvalidierte deutsche Übersetzung)

\begin{tabular}{|c|c|c|c|c|c|}
\hline & 0 & 1 & 2 & 3 & 4 \\
\hline Zeit Stuhlgang (min) & $\leq 5$ & $6-10$ & $11-20$ & $21-30$ & $>30$ \\
\hline $\begin{array}{l}\text { Defäkationsversuche pro } \\
\text { Tag }\end{array}$ & 1 & 2 & $3-4$ & $5-6$ & $>6$ \\
\hline $\begin{array}{l}\text { Anale/vaginale Digitati- } \\
\text { on }\end{array}$ & Nie & $\begin{array}{l}>1 / \text { Monat }<1 / \\
\text { Woche }\end{array}$ & $\begin{array}{l}\text { 1-mal/ } \\
\text { Woche }\end{array}$ & $\begin{array}{l}\text { 2- bis 3-mal/ } \\
\text { Woche }\end{array}$ & Immer \\
\hline Laxanzien & Nie & $\begin{array}{l}>1 / \text { Monat }<1 / \\
\text { Woche }\end{array}$ & $\begin{array}{l}\text { 1-mal/ } \\
\text { Woche }\end{array}$ & $\begin{array}{l}\text { 2- bis 3-mal/ } \\
\text { Woche }\end{array}$ & Immer \\
\hline Klysmen & Nie & $\begin{array}{l}>1 / \text { Monat }<1 / \\
\text { Woche }\end{array}$ & $\begin{array}{l}\text { 1-mal/ } \\
\text { Woche }\end{array}$ & $\begin{array}{l}\text { 2- bis 3-mal/ } \\
\text { Woche }\end{array}$ & Immer \\
\hline Inkomplette Defäkation & Nie & $\begin{array}{l}>1 / \text { Monat }<1 / \\
\text { Woche }\end{array}$ & $\begin{array}{l}\text { 1-mal/ } \\
\text { Woche }\end{array}$ & $\begin{array}{l}\text { 2- bis 3-mal/ } \\
\text { Woche }\end{array}$ & Immer \\
\hline Pressen bei Defäkation & Nie & $\begin{array}{l}>1 / \text { Monat }<1 / \\
\text { Woche }\end{array}$ & $\begin{array}{l}\text { 1-mal/ } \\
\text { Woche }\end{array}$ & $\begin{array}{l}\text { 2- bis 3-mal/ } \\
\text { Woche }\end{array}$ & Immer \\
\hline Stuhlkonsistenz & Weich & Hart & Hart, wenig & Koprolith & \\
\hline
\end{tabular}

Tab. 2 St. Mark's Incontinence Score. (Mod. nach [10], nichtvalidierte deutsche Übersetzung)

\begin{tabular}{|c|c|c|c|c|c|}
\hline & Nie & Selten & Manchmal & Wöchentlich & Täglich \\
\hline Inkontinenz für festen Stuhl & 0 & 1 & 2 & 3 & 4 \\
\hline Inkontinenz für flüssigen Stuhl & 0 & 1 & 2 & 3 & 4 \\
\hline Inkontinenz für Gase & 0 & 1 & 2 & 3 & 4 \\
\hline $\begin{array}{l}\text { Einschränkung der Lebensge- } \\
\text { wohnheiten }\end{array}$ & 0 & 1 & 2 & 3 & 4 \\
\hline \multicolumn{4}{|l|}{-} & Nein & $J a$ \\
\hline \multicolumn{4}{|c|}{ Tragen von Vorlagen oder analen Tampons } & 0 & 2 \\
\hline \multicolumn{4}{|c|}{ Einnahme stuhleindickender Medikation } & 0 & 2 \\
\hline \multicolumn{4}{|c|}{ Unvermögen, den Stuhlgang für 15 min zu unterdrücken } & 0 & 4 \\
\hline \multicolumn{6}{|c|}{$\begin{array}{l}\text { Nie keine Episode in den letzten } 4 \text { Wochen, Selten } 1 \text { Episode in den letzten } 4 \text { Wochen, Manchmal }>1 \\
\text { Episode in den letzten } 4 \text { Wochen, aber }<1 \text { pro Woche, Wöchentlich } \geq 1 \text { Episode pro Woche, aber }<1 \\
\text { pro Tag, Täglich } \geq 1 \text { Episode pro Tag }\end{array}$} \\
\hline
\end{tabular}

Score nach Altomare, der anhand von 6 Parametern (Dauer, Anzahl der Defäkationsversuche, Notwendigkeit von Entleerungshilfen, das Gefühl der unvollständigen Entleerung, Pressen bei Defäkation und die Stuhlkonsistenz) den Schweregrad der Obstruktion von 0 bis 31 angibt (- Tab. 1; [8]).

Es empfiehlt sich, bei funktioneller Enddarmentleerungsstörung immer beide Formen der Funktionseinschränkung $\mathrm{zu}$ erfassen: Kontinenz und OD, die oft miteinander assoziiert sind. So leiden ca. $46 \%$ der Patienten mit einer OD an einer FI und $51 \%$ der Patienten mit FI fühlen sich nie vollständig entleert [9]. Ebenso kann die Therapie der einen Symptomatik zu einer Verschlechterung der anderen führen. Scores der FI sind beispielsweise der häufig verwendete Cleveland Clinic Incontinence Score nach Wexner, der validierte FISI-Score nach Rockwood oder der St. Mark's Incontinence Score (• Tab. 2; [10]).

Liegt eine funktionelle Einschränkung im Sinne einer OD oder FI vor, so folgt in der klinisch-proktologischen Untersuchung die Darstellung eines möglichen morphologischen Korrelats der Beschwerden: die Intussuszeption und/ oder die komplexe Rektozele. Bereits hier können eine zugrunde liegende Beckenbodensenkung, eine anteriore Rektozele und in der Rektoskopie eine Intussuszeption diagnostiziert werden, ebenso wie Senkungen der anderen Kompartimente.

Morphologische Veränderungen aller Kompartimente können auch in einer dynamischen Defäkographie dargestellt werden. Hier können Intussuszeption, Rektozele, Enterozele oder Vesikozele und deren Ausmaß wie auch eine unvollständige Entleerung verifiziert werden, deren Vorliegen sowohl für die
Indikation als auch für die Verfahrenswahl von Bedeutung sein kann. Da bei morphologischer OD mit funktioneller Einschränkungen der Lebensqualität nur eine relative Operationsindikation besteht, ist auch aus medikolegalen Gründen die Dokumentation über die Darstellung von morphologischen Veränderungen (Defäkographie) ebenso wie die Dokumentation über das Vorliegen und das Ausmaß der berichteten funktionellen Einschränkung (Scores) von hoher Relevanz. Da zumeist komplexe Veränderungen und Funktionseinschränkungen verschiedener Kompartimente bei Beckenbodensenkung vorliegen, ist eine interdisziplinäre Fallbesprechung zusammen mit der Gastroenterologie und Urogynäkologie wünschenswert.

\section{Kontraindikationen und alternative Verfahrenswahl}

Nach Stellen der Operationsindikation müssen im nächsten Schritt absolute und relative Kontraindikationen abgeklärt, alternative Verfahren abgewogen und mit dem Patienten besprochen werden. Je besser der Patient über seine Erkrankung informiert und über seine Befunde, operative Risiken und Therapiealternativen aufgeklärt wird, desto eher können realistische Therapieerwartungen erzielt werden.

Kontraindikation zur Netzimplantation ventral des Rektums besteht bei Vorliegen einer aktiven Proktitis bzw. einer chronisch-entzündlichen Darmerkrankung. Relative Kontraindikation stellen Frauen mit noch nicht abgeschlossener Familienplanung dar, das Vorliegen einer Endometriose des Douglas oder vorangegangene Schübe einer Sigmadivertikulitis. Ebenfalls eng zu stellen ist die Indikation bei mehrfachen abdominellen Voroperationen oder vorangegangener Radiatio des kleinen Beckens. Zur Therapie einer morphologischen OD sollten zuvor konservativ behandelbare Erkrankungen wie die funktionelle OD (Beckenbodendyssynergie) sowie auch eine Slow-transit-Konstipation mit einer den gesamten Dickdarm betreffenden verlangsamten Passage ausgeschlossen werden. Auch bei Vorliegen signifikanter psychiatrischer Vorerkrankungen, 
chronischer Schmerzsyndrome oder Reizdarmsyndrom kann das postoperative Ergebnis nach LVR limitiert sein, auch wenn dazu kaum Daten vorliegen $[11,12]$.

\section{》) Transanale Techniken können eine Alternative sein}

Als alternative Verfahren sind insbesondere transanale Techniken zu nennen: bei Vorliegen eines externen Rektumvollwandprolaps die Mukosaresektion nach Rehn-Delorme und die Rektosigmoidresektion nach Altemeier, im Fall einer morphologischen OD die transanale Rektumresektion mit einem Klammernahtinstrument („stapled transanal rectal resection“, STARR, Contour ${ }^{\circledR}$ $\operatorname{Transtar}^{\mathrm{TM}}$ ), wobei die Anwendung rückläufig ist und die Resektionsrektopexie als alternatives abdominelles Verfahren angewandt wird, die jedoch in Europa der LVR gewichen ist [2, 3]. Auf die alternativen Operationsmethoden wird in der Diskussion eingegangen. Da kaum vergleichende Daten existieren, kann kein evidenzbasierter Therapiealgorithmus gegeben werden, wann welches Verfahren zum Einsatz kommen sollte.

\section{Wichtige Aspekte der Aufklärung}

Insbesondere bei Vorliegen einer relativen Operationsindikation bei morphologischer OD kommt dem „shared decision making“, also der gemeinsamen Entscheidungsfindung mit einem informierten, selbstbestimmten Patienten eine Schlüsselrolle zu. Diese setzt wiederum ein sorgfältiges Aufklärungsgespräch mit dem Patienten über seine Erkrankung, Interpretation der erhobenen Befunde, Bandbreite der Therapiealternativen und die einzelnen Operationsverfahren mit ihren Ergebnissen, Risiken und Langzeitfolgen voraus.

Dazu zählen Persistenz oder Rezidiv der Beschwerden bzw. der Morphologie, das Auftreten von De-novo-Beschwerden (OD oder FI) ebenso wie postoperativ funktionelle Veränderungen der anderen Kompartimente mit einer mögli-

coloproctology 2020 · 42:331-338 https://doi.org/10.1007/s00053-020-00457-6

๑) Springer Medizin Verlag GmbH, ein Teil von Springer Nature 2020

\section{Kim}

\section{Laparoskopische ventrale Rektopexie}

\section{Zusammenfassung}

Die laparoskopische ventrale Netzrektopexie ist ein Operationsverfahren, das 2004 erstmalig von D'Hoore und Penninckx beschrieben wurde. Die niedrige Morbidität und die guten funktionellen Ergebnisse haben dazu geführt, dass sie seit ihrer Einführung zu dem führenden operativen Verfahren in Europa zur Korrektur eines externen Rektumprolapses wie auch des inneren Rektumprolapses (Intussuszeption) bei funktioneller Enddarmentleerungsstörung avanciert ist. Seltene, jedoch zum Teil schwerwiegende netzassoziierte Komplikationen wie Erosionen und Infekte können aufwändige Revisionen zur Folge haben und machen insbesondere unter Berücksichtigung der benignen Grunderkrankung eine kritische Überprüfung der operativen Indikationsstellung, eine sorg- fältige Patientenselektion und -aufklärung im Sinne eines "shared decision making" als entscheidende Schritte einer erfolgreichen Therapie notwendig. In diesem Beitrag wird eine Operationsmethode vorgestellt, die Schritt für Schritt das Vorgehen bei der laparoskopischen ventralen Netzrektopexie darstellt. Zusätzlich werden das perioperative Management, Ergebnisse und die aktuelle Literatur diskutiert. Zur Illustration wird eine robotische ventrale Netzrektopexie bei obstruktiver Defäkation auf dem Boden einer Intussuszeption vorgestellt.

\section{Schlüsselwörter}

Netzrektopexie - Externer Rektumprolaps Obstruktive Defäkation - Intussuszeption . Rektozele

\section{Laparoscopic ventral rectopexy}

\section{Abstract}

The laparoscopic ventral mesh rectopexy represents an operative approach first described by $\mathrm{D}^{\prime}$ Hoore and Penninckx in 2004. Due to low morbidity rates and good functional results it has become the most widely accepted surgical approach throughout Europe since its introduction for the treatment of external rectal prolapse and internal rectal prolapse (intussusception) with functional rectal emptying disorders. Rare, but sometimes severe mesh-associated complications, such as erosions and infections can lead to complicated revision surgery. Bearing the benign underlying disease in mind, they make a critical review of the indications for surgery, stringent patient selection and clarification in the sense of shared decision making, crucial steps for successful treatment. In this article the technical approach for laparoscopic ventral mesh rectopexy is described step by step. Additionally, perioperative management, outcome and the current literature are discussed. The report is illustrated with images of robotic ventral mesh rectopexy in obstructive defecation due to intussusception.

\section{Keywords}

Laparoscopic ventral mesh rectopexy . External rectal prolapse - Obstructive defecation · Intussusception - Rectocele chen Veränderung der Blasen- oder Sexualfunktion.

Perioperative Komplikationen betreffen die Konversion, Verletzung des Plexus hypogastricus, der V. iliaca communis, des rechtsseitigen Ureters, der vaginalen Hinterwand oder des Rektums. Besonderes Augenmerk liegt auf sehr selten auftretenden Netzkomplikationen, zu denen Netzerosionen und Netzinfekte zählen und in einigen Fällen aufwändige Revisionen zur Folge haben können [5].

\section{Operationsverfahren}

\section{Vorbereitung, Lagerung und Zugangswege}

Der Patient wird mit 2 Klysmen am Operationstag abgeführt und erhält einen transurethralen Dauerkatheter wie auch eine antibiotische Single-shot-Prophylaxe vor Hautschnitt. Anschließend wird der Patient in flacher Steinschnittlage mit beidseits angelegten Armen und Schulterstützen positioniert. 


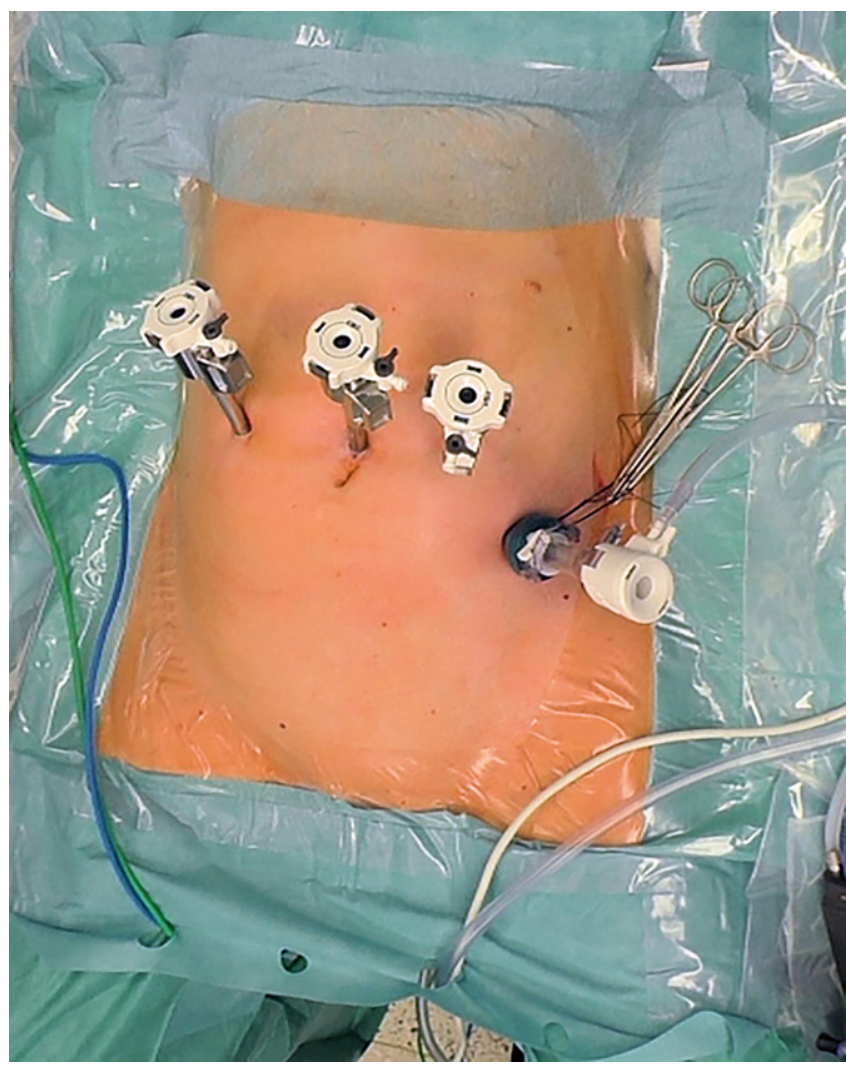

Abb. 1 A Patientenlagerung in flacher Steinschnittlage mit Trokarpositionierungen für eine robotisch unterstützte ventrale Netzrektopexie

Beim konventionell-laparoskopischen Vorgehen stehen Operateur und Assistent rechts vom Patienten und die Instrumentierpflegekraft links, ein Monitor ist über dem linken Bein des Patienten positioniert. Konventionelllaparoskopisch erfolgt die Trokarpositionierung mit der Optik supraumbilikal, einem 5-mm-Trokar im linken Unterbauch und einem 12-mm-Trokar im rechten Unterbauch.

Beim robotischen Vorgehen mit dem da Vinci Xi ${ }^{\circledR}$ System (Intuitive Surgical ${ }^{\circledR}$, Sunnycale, CA, USA) ist das Vorgehen im Hause die Positionierung des Assistenten und der Instrumentierpflegekraft links des Patienten und des Roboters rechts mit Einstellung des Beckens. Ein 12-mm-Assistenten-Trokar wird beim robotischen Vorgehen im linken Unterbauch eingebracht und drei 7-mm-Trokare auf einer Linie supraumbilikal für das Endoskop und jeweils einer links bzw. rechts davon. Der vierte Roboterarm wird dabei nicht verwendet (• Abb. 1).

\section{Präparation}

Der Patient wird in Kopftieflage verbracht, sodass der Dünndarm problemlos aus dem kleinen Becken nach kranial geschlagen werden kann. Bei Frauen und noch vorhandenem Uterus kann die Fixierung des Uterus ventral an die Bauchdecke für die weitere Präparation von Vorteil sein. Dazu wird mit einer doppelarmierten geraden Nadel durch die Bauchdecke eingestochen, anschließend ein Ligamentum latum uteri umfahren und mit der Nadel wieder durch die Bauchdecke ausgestochen, bevor die Naht vor der Bauchdecke verknotet wird.

Nun erfolgt die Identifikation des Promontoriums rechtsseitig als späterer Verankerungspunkt des Netzes (- Abb. 2). Nach Identifikation des Promontoriums wird eine J-förmige Inzision des Peritoneums mit der monopolaren Schere durchgeführt, beginnend rechts am Promontorium, nach kaudal pararektal rechts fortgeführt und anschließend bogenförmig von rechts nach links über dem Douglas-Raum bei Frauen vervollständigt (•Abb. 3). Dabei wird der rechtsseitige Ureter an der Beckenachse identifiziert und geschont, ebenso wie die V. iliaca communis bzw. externa.

Nun beginnt die Präparation, bei der im Septum rectovaginale bei weiblichen Patienten ventral des Rektums bis auf den Beckenboden vorgegangen wird. Ziel ist es, dass der Beckenboden links und rechts ventral am Rektum deutlich erkennbar wird. Auch die Präparation unmittelbar ventralseitig sollte bis auf den Beckenboden erfolgen, um so das Netz unterhalb einer anterioren Rektozele platzieren zu können (• Abb.4). Dabei kann ein durch einen zweiten Assistenten transvaginal eingebrachtes Spekulum oder einen Stieltupfer die Vagina nach ventral aufgerichtet werden, um so die Dissektion nach kaudal im Septum rectovaginale zu erleichtern. Die Schonung der dünnen vaginalen Hinterwand bei zumeist postmenopausalen Frauen und der ventralen Rektumwand muss beachtet werden. Die ausreichende Präparation nach kaudal auf den Beckenboden kann abschließend digital-rektal überprüft werden.

\section{Netzimplantation}

Nun wird ein Netz mit einer Länge von ca. $15 \mathrm{~cm}$ und einer Breite von ca. $4 \mathrm{~cm}$ mit nach links ausladendem Kopf angepasst an die Patientengröße und den -si- 


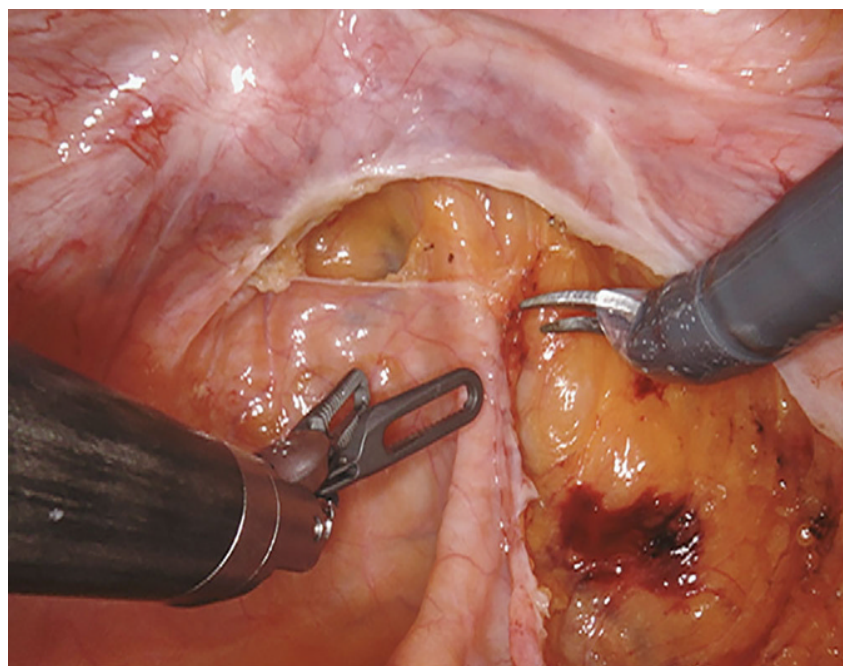

Abb. $3 \Delta$ Erweiterung der peritonealen Inzision von pararektal rechts über dem Douglas, Hockeyschläger-förmig nach links

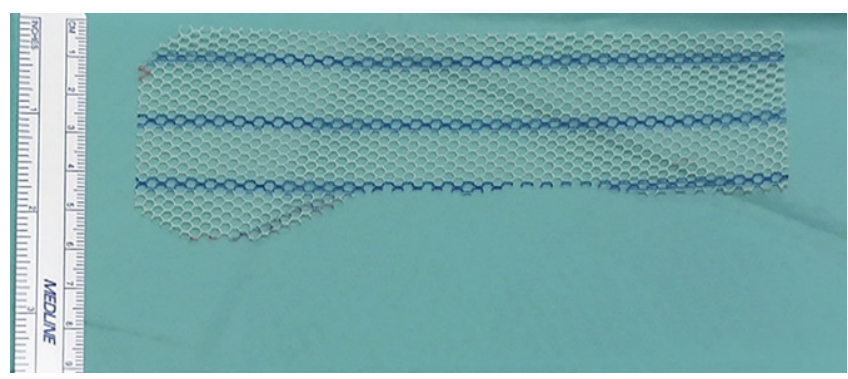

Abb. $5 \Delta$ Implantation eines ca. $4 \mathrm{~cm}$ breiten und ca. $15 \mathrm{~cm}$ langen Netzstreifens mit nach links ausladendem Kopf

tus über den 12-mm-Trokar eingebracht (• Abb.5). Die Positionierung des Netzes sollte soweit wie möglich nach kaudal erfolgen und entweder mit langsam resorbierbaren Nähten an der Rektumvorderwand (z. B. PDS $\left.{ }^{\circledR} 2-0\right)$ oder zusätzlich mit nichtresorbierbaren Nähten streng am Beckenboden (z.B. Ethibond ${ }^{\circledR}$ 2-0) fixiert werden. Das Vorgehen im Hause beinhaltet 4 Nähte pararektal am Beckenboden, jeweils 2 links und 2 rechts sowie 2-3 lockere Nähte ventralseitig an der Tunica adventitia des Rektums (• Abb. 6). Bei der Fixierung am Rektum dürfen keine transmuralen Stiche durchgeführt werden, um so die Gefahr von Netzkomplikationen zu vermeiden. In Abhängigkeit des Befundes werden die Stiche im Bereich des Rektums durchgeführt, zusätzliche Stiche können zur Fixierung der posterioren Fornix uteri am Netz zur Ver-

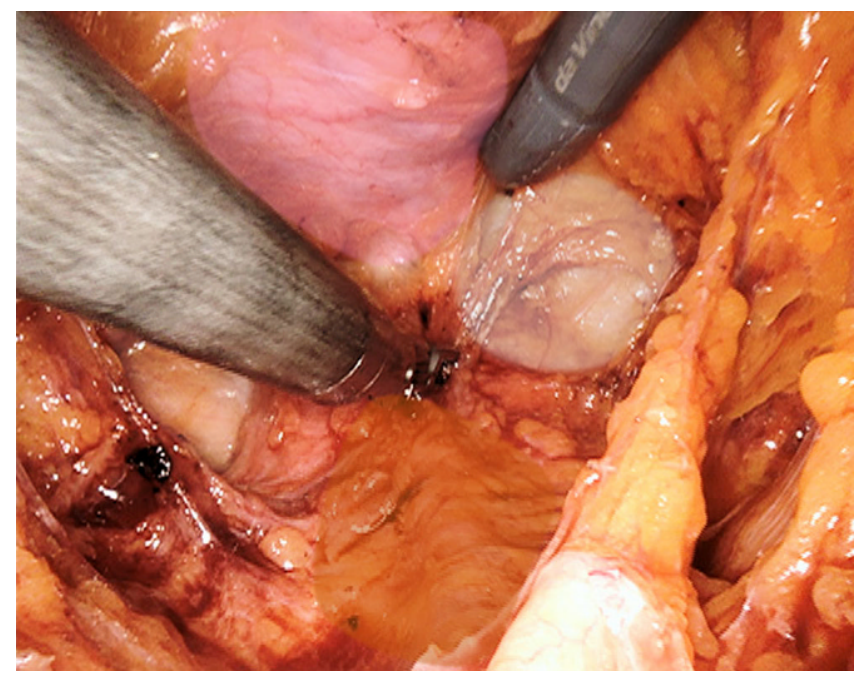

Abb. 4 \ Präparation des Septum rectovaginale der Frau unter Schonung der vaginalen Hinterwand (rosa unterlegt) bis zur Darstellung des Beckenbodens ventralseitig rechts und links (grau unterlegt) des Rektums (braun unterlegt)

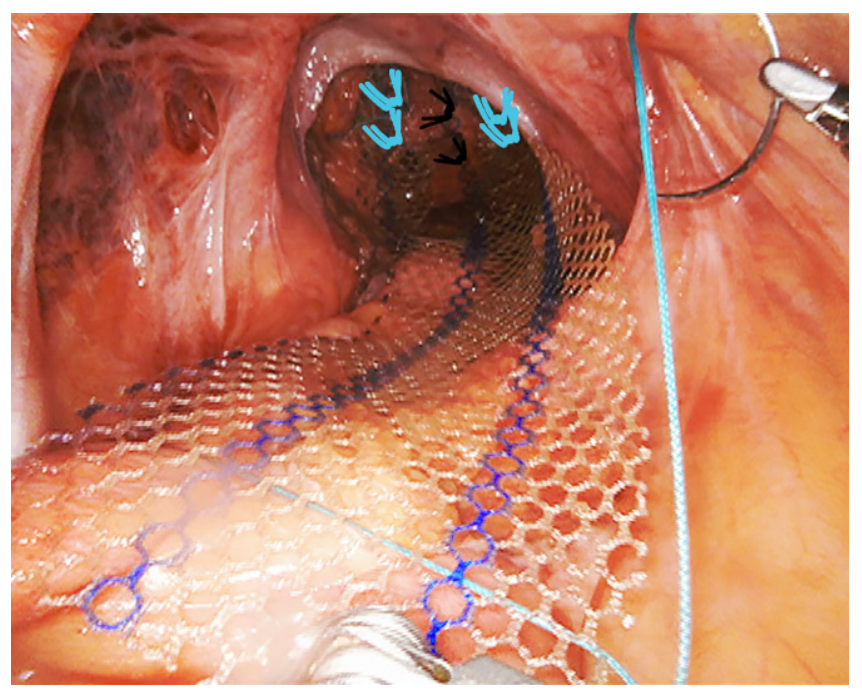

Abb. $6 \Delta$ Fixierung des Netzes am Beckenboden beidseits mit nichtresorbierbarem Nahtmaterial (türkis unterlegt) und an der ventralen Seite des Rektums mit resorbierbarem Nahtmaterial (blau unterlegt)

meidung einer Enterozele durchgeführt werden.

Anschließend erfolgt die Fixierung des kranialen Netzanteils am Promontorium spannungsfrei und überstehende Netzanteile können eingekürzt werden, falls erforderlich. Eine genaue Angabe zur Spannung existiert nicht, es sollte jedoch bedacht werden, dass es nach Ablassen des Pneumoperitoneums zu Veränderungen von Spannungsverhältnissen kommen kann. Die Fixierung am Promontorium erfolgt mit 2 Stichen mittels nichtresorbierbarem Nahtmaterial (z.B. Ethibond ${ }^{\circledR}$ der Stärke 0) oder Tackern (• Abb. 7). Auf eine Verletzung der V. iliaca communis und eine Beeinträchtigung des Plexus hypogastricus zur Vermeidung einer postoperativen Konstipation muss geachtet werden.

Nachfolgend wird das Peritoneum fortlaufend mit resorbierbarem, multifilamentem Nahtmaterial verschlossen, sodass das Netz vollständig mit Peritoneum bedeckt ist (• Abb. 8). 


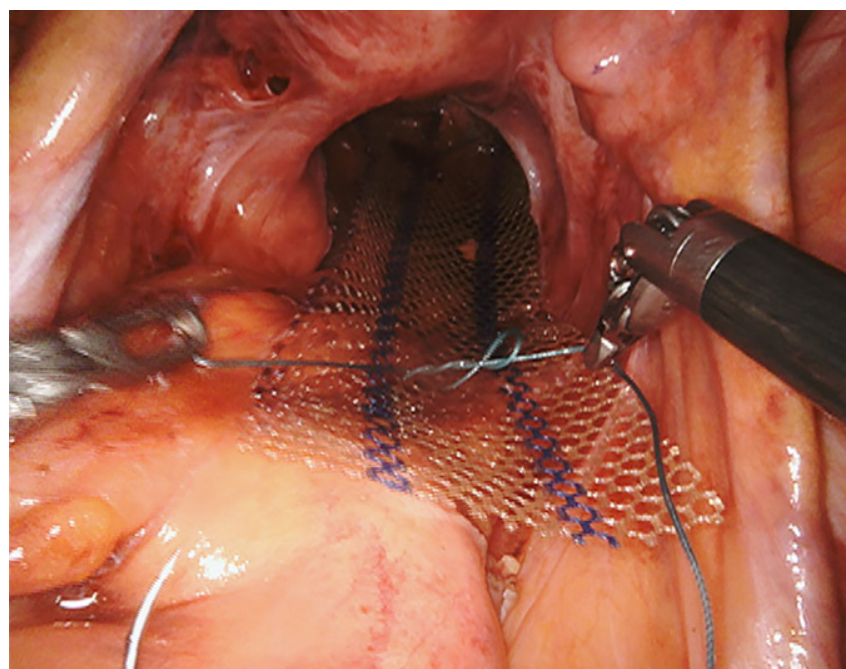

Abb. 7 ^ Spannungsfreie Lage des Netzes und Naht des Netzes am Promontorium

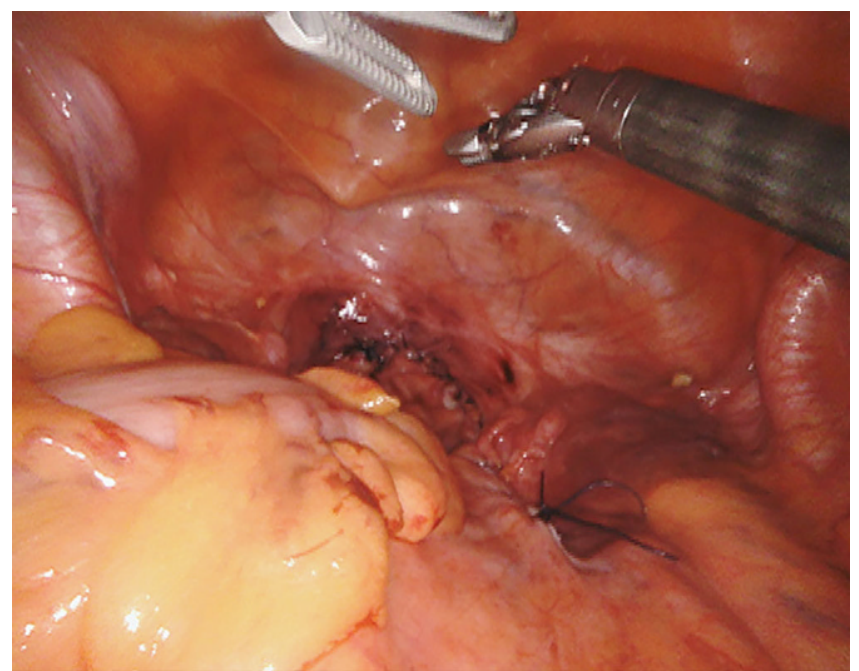

Abb. 8 A Abschließender Verschluss des Peritoneums, sodass keine Netzanteile freiliegen
Es erfolgt der Faszienverschluss des 12-mm-Zugangs. Die Annaht des Uterus, falls zuvor durchgeführt, wird gelöst und der transurethrale Dauerkatheter entfernt.

Postoperativ können die umgehende Mobilisation und der Kostaufbau erfolgen. Stuhlregulierende Maßnahmen, z. B. in Form von Macrogol werden für ca. 4-6 Wochen empfohlen.

\section{Ergebnisse}

Insgesamt ist die Evidenz zur LVR niedrig bis sehr niedrig: Es existieren kaum vergleichende oder randomisierte Studien. Die Interpretation der Daten ist eingeschränkt aufgrund der Heterogenität der Studien in Hinblick auf unterschiedlich angewandte Netzarten, Netzfixationen, Kombination mit anderen operativen Verfahren, verschiedene Indikationen (externer Rektumprolaps vs. morphologische OD oder FI) und unterschiedlichen Definitionen eines Rezidivs (morphologisch vs. symptomatisch).

Unter Beachtung dessen kommt es postoperativ nach einem Follow-up von 19 Monaten bei 74\% (95\% Konfidenzintervall [KI] 66-82) der Patienten zu einer Verbesserung der Entleerungsstörung nach LVR und bei $60 \%$ zu einer Verbesserung der FI bei deutlicher Varianz (95\% KI 45-75). Zu dem Auftreten einer De-novo-OD oder einer -FI liegen kaum Daten vor. In einer Metaanalyse wird die Rate an De-novo-OD oder Verschlechterung einer OD mit 4,5\% (:Range 2,4-11,5\%) angegeben und die der De-novo-FI mit 7,5\% (Range: $3,1-14,5 \%)$ bei einer Rezidivrate von 5,8\% (95\% KI 4,2-7,5) nach 19 Monaten im Median [14]. Für Langzeitfolgen der Netzimplantation in Hinblick auf eine sexuelle Dysfunktion liegen nahezu keine Daten vor, postoperative Schmerzsyndrome können bei 2,9\% der Patienten auftreten [5].

Insgesamt ist die LVR von einer niedrigen Gesamtmorbidität von 5-15\% gekennzeichnet, Netzkomplikationen sind selten und mit $0-3,9 \%$ beschrieben, die in einer großen zeitlichen Varianz von 2 bis zu 78 Monate postoperativ auftreten können $[5,12]$. Auch wenn netzassoziierte Komplikationen eine seltene Entität darstellen, können diese in $40 \%$ der Fälle herausfordernde abdominelle Revisionen zur Folge haben, die in seltenen Situationen wie einer rektovaginalen Fistulierung auch eine ultratiefe Rektumresektion unter Stomaprotektion notwendig machen können [5].

\section{Diskussion}

Auch wenn die funktionellen Ergebnisse der LVR sehr gut sind, so ist zu beachten, dass diese zumeist auf Beobachtungsstudien niedriger Evidenz beruhen mit limitiertem Follow-up. Trotz anatomi- scher Korrektur der morphologischen Veränderungen kann nicht immer von einer Heilung der funktionellen Einschränkung ausgegangen werden, sodass mit dem Patienten präoperativ eine realistische Erwartungshaltung besprochen werden sollte.

\section{》) Die Netzkomplikationsrate beträgt maximal $4 \%$}

Aktuell rücken insbesondere durch Patientengruppen Folgeerscheinungen nach Netzimplantation im kleinen Becken in den Fokus. Auch wenn die Rate an Netzerosionen und-komplikationen nicht mit denen transvaginal eingebrachter Netze vergleichbar ist, so gilt bei der Indikation zur Implantation eines Fremdkörpers besondersim Rahmen einer funktionellen, benignen Erkrankung besondere Sorgfalt. Die Netzkomplikationsrate beträgt im Worst-case-Szenario $4 \%$ und ist damit niedrig. Netzkomplikationen wie auch Rezidive können bei einliegendem Netz jedoch zu aufwändigen Revisionseingriffen bei gutartiger Grunderkrankung führen, wobei ca. 8-9\% der Patienten nach LVR einem Revisionseingriff im Verlauf unterzogen werden $[4,5]$.

$\mathrm{Ob}$ es durch die Nutzung eines speziellen Netzes, sei es biologisch oder synthetisch, zu einer veränderten Morbidität kommt, kann abschließend nicht beantwortet werden. Die aktuelle Datenlage 
kann keinen Unterschied hinsichtlich Rezidiven, Funktion oder Netzkomplikationen festmachen. Weitere, insbesondere komparative Daten mit einem längeren Follow-up sind hier notwendig.

Vor diesem Hintergrund sollten Therapiealternativen ausgewogen mit dem Patienten diskutiert werden: neben der konservativen Therapie die transanale Technik und andere abdominelle Verfahren wie die Resektionsrektopexie. Letztere scheint mit einer höheren Rate an Komplikationen nach Resektion und Anastomosierung im Vergleich zur LVR einherzugehen [13]. Der Eingriff nach Rehn-Delorme und die AltemeierResektion als transanale Verfahren zur Therapie des externen Rektumvollwandprolapses stellen sinnvolle Alternativen dar, die möglicherweise eine höhere Rezidivrate als die LVR aufweisen, auch wenn dazu keine vergleichenden Daten vorliegen. Kommt es zu einem externen Vollwandrezidiv, so sind solche nach transanalem Vorgehen möglicherweise einer Revision leichter zugänglich als nach Netzimplantation ventral des Rektums, deren Lage sowohl eine abdominelle Revision als auch eine transanale Resektion erschweren kann. Als transanale Verfahren bei der morphologischen OD gelten STARR oder der Contour $^{\circledR}$ Transtar $^{\mathrm{TM}}$. Deren Anwendung hat jedoch deutlich abgenommen, mutmaßlich aufgrund von De-novo-FI und Urge-Symptomen wie auch nachlassendem Effekt im Langzeit-Follow-up [3]. Nichtsdestotrotz können transanale Klammernahtverfahren insbesondere bei isolierter OD ohne Vorliegen einer Urge- oder FI-Symptomatik und ohne ausgeprägten Beckenbodentiefstand und nach Diskussion mit dem Patienten in Betracht gezogen werden. Vor dem Hintergrund der existierenden Evidenz niedriger Qualität kann jedoch kein operativer Therapiealgorithmus gegeben werden.

Die sorgfältige Patientenselektion auf dem Boden einer differenzierten Diagnostik, die detaillierte Aufklärung des Patienten über seine Erkrankung, die diagnostischen Befunde und die Diskussion von Therapiealternativen mit den jeweiligen Risiken, Folgen und realistischen funktionellen Verbesserungen im
Sinne eines "shared decision making“ sind die maßgeblichen Erfolgsfaktoren bei der Anwendung der LVR.

\section{Fazit für die Praxis}

- Der Erfolg der LVR hängt neben der operativen Prozedur maßgeblich von der differenzierten Indikationsstellung, insbesondere bei funktioneller Entleerungsstörung, ab.

- Das Ausmaß der Funktionseinschränkung sollte anhand von validierten Messinstrumenten festgehalten und ein morphologisches Korrelat als Ansatz jedweder operativen Korrektur in der klinisch-proktologischen Untersuchung und einer Defäkographie dargestellt werden.

- Die funktionellen Ergebnisse der LVR sind sehr gut, die Morbidität gering bei jedoch niedriger Evidenz der Datenlage.

- Dennoch müssen Patienten insbesondere über mögliche Langzeitfolgen einer selten auftretenden Netzkomplikation im kleinen Becken aufgeklärt und Alternativen mit ihnen diskutiert werden.

- Unter Beachtung dessen stellt die LVR bei sorgfältiger Indikationsstellung ein sicheres Verfahren mit guten funktionellen Ergebnissen dar.

Porrespondenzadresse
Klinik und Poliklinik für
Allgemein-, Viszeral-,
Transplantations-, Gefäß-
und Kinderchirurgie,
Universitätsklinikum
Würzburg
Oberdürrbacher Straße 6,
97080 Würzburg,
Deutschland
Kim_M@ukw.de

\section{Einhaltung ethischer Richtlinien}

Interessenkonflikt. M. Kim gibt an, dass kein Interessenkonflikt besteht.

Für diesen Beitrag wurden von den Autoren keine Studien an Menschen oder Tieren durchgeführt. Für die aufgeführten Studien gelten die jeweils dort angegebenen ethischen Richtlinien.

\section{Literatur}

1. D'Hoore A, Cadoni R, Penninckx F (2004) Longterm outcome of laparoscopic ventral rectopexy for total rectal prolapse. Br J Surg 91:1500-1505

2. Formijne Jonkers HA, Draaisma WA, Wexner SD et al (2013) Evaluation and surgical treatment of rectal prolapse: an international survey. Colorectal Dis 15:115-119

3. Kim M, MeuretteG, Ragu R, LehurPA (2016) Current surgical treatment of obstructed defecation among selected European opinion leadersin pelvic floor surgery. Tech Coloproctol 20(6):395-399. https://doi.org/10.1007/s10151-016-1473-z

4. Consten EC, van lersel JJ, Verheijen PM, Broeders IA, Wolthuis AM, D'Hoore A (2015) Long-term outcome after laparoscopic ventral mesh rectopexy: an observational study of 919 consecutive patients. Ann Surg 262(5):742-747. https://doi. org/10.1097/SLA.0000000000001401 (discussion 747-8)

5. Evans C, Stevenson AR, Sileri P, Mercer-Jones MA, Dixon AR, Cunningham C, Jones OM, Lindsey I (2015) A multicenter collaboration to assess the safety of laparoscopic ventral rectopexy. Dis Colon Rectum 58(8):799-807. https://doi.org/10.1097/ DCR.0000000000000402

6. Cavallaro PM, Staller K, Savitt LR, Milch H, Kennedy K, Weinstein MM, Ricciardi R, Bordeianou LG (2019) The contributions of internal intussusception, irritable bowel syndrome, and pelvic floor dyssynergia to obstructed defecation syndrome. Dis Colon Rectum 62(1):56-62. https://doi.org/10. 1097/DCR.0000000000001250

7. Palit S, Bhan C, Lunniss PJ, Boyle DJ, Gladman MA, Knowles CH, Scott SM (2014) Evacuation proctography: a reappraisal of normal variability. Colorectal Dis 16(7):538-546. https://doi.org/10. 1111/codi.12595

8. Altomare DF, Spazzafumo L, Rinaldi M, Dodi G, Ghiselli R, Piloni V (2008) Set-up and statistical validation of a new scoring system for obstructed defaecation syndrome. Colorectal Dis 10(1):84-88

9. Nurko S, Scott SM (2011) Coexistence of constipation and incontinence in children and adults. Best Pract Res Clin Gastroenterol 25(1):29-41. https:// doi.org/10.1016/j.bpg.2010.12.002

10. Maeda Y, Parés D, Norton C, Vaizey CJ, Kamm MA (2008) Does the St. Mark's incontinence score reflect patients' perceptions? A review of 390 patients. Dis Colon Rectum 51(4):436-442. https:// doi.org/10.1007/s10350-007-9157-4

11. Knowles CH, Grossi U, Horrocks EJ, Pares D, Vollebregt PF, Chapman M, Brown S, Mercer-Jones M, Williams AB, Yiannakou Y, Hooper RJ, Stevens N, Mason J, NIHR CapaCiTY working group, Pelvic floor Society, European Society of Coloproctology (2017) Surgery for constipation: systematic review and practice recommendations: graded practice and future research recommendations. Colorectal Dis 19(3):101-113. https://doi.org/10.1111/codi. 13775

12. Mercer-Jones MA, D'Hoore A, Dixon AR, Lehur $P$, Lindsey I, Mellgren A, Stevenson AR (2014) Consensus on ventral rectopexy: report of a panel of experts. Colorectal Dis 16(2):82-88. https://doi. org/10.1111/codi.12415

13. Grossi U, Knowles CH, Mason J, Lacy-Colson J, Brown SR, NIHR CapaCiTY working group, Pelvic floor Society (2017) Surgery for constipation: systematic review and practice recommendations: results II: hitching procedures for the rectum (rectal suspension). Colorectal Dis 19(3):37-48. https:// doi.org/10.1111/codi.13773 


\section{How I do it}

14. Emile SH, Elfeki HA, Youssef M, Farid M, Wexner SD (2017) Abdominal rectopexy for the treatment of internal rectal prolapse: a systematic review and meta-analysis. Colorectal Dis 19(1):013-024. https://doi.org/10.1111/codi.13574

Hier steht eine Anzeige.

\section{Springer}

